\title{
¿INCLUIR LA OBESIDAD EN EL MANUAL DE ENFERMEDADES MENTALES (DSM-IV)?
}

\section{OBESITY AS A MENTAL DISORDER IN DSM-IV?}

\author{
Patricia Cordella M. \\ Unidad Trastornos de Alimentación, Departamento de Psiquiatría \\ Pontificia Universidad Católica de Chile
}

\begin{abstract}
The main objective of this study is to expand the observation of the relationship between eating (ingestion) and emotions in obesity. Cerebral regions that share primary circuits in both eating and emotions were analyzed. It is proposed that eating is more than the act of feeding and includes other mental function activities such as self regulation and pleasure seeking, and has a role in the emotional adjustment of significant relationships (family, couple). It is proposed that if obesity could be included in DSM, new treatment and prevention alternatives could be developed.
\end{abstract}

Key words: obesity, mental disorder, emotional alterations, DSM.

Este trabajo fue recibido el 30 de Enero de 2008 y aceptado para ser publicado el 19 de Agosto de 2008.

\section{MANUAL DSM}

El DSM-IV (manual diagnóstico y estadístico de enfermedades mentales, versión número cuatro) (1) se encarga de delimitar las patologías mentales a través de un método que junta estadísticamente frecuencia, intensidad y pronóstico de enfermedades creando y dando nombre proprio a conjuntos de síntomas y signos. Esta constitución establece los límites del estado patológico de la mente y por tanto demarca los campos de la competencia psiquiátrica dentro del quehacer de la medicina.

Siguiendo un modelo de agrupaciones probables determina configuraciones fisiológicas de frecuente aparición que pueden ser nominadas en consenso y tratadas en forma común. Para la especialidad psiquiátrica la enfermedad se refiere a alteraciones tanto conductuales de los pacientes como de la vivencia emocional. Los órganos, aunque participantes activos de ciertas patologías no se reconocen como asiento patológico, excepto el sistema nervioso central y autónomo.

Se acepta un orden, se delimitan los administradores y los territorios nosológicos, delegándose la cura (remoción de la causa) a quien corresponda. Así ocurre que cada enfermedad pasa a formar parte del patrimonio de una especialidad, quienes la toma a cargo: la estudian y le buscan soluciones.

Sucesivas investigaciones y las reflexiones que derivan de ellas van cargando de significado la patología y van generando un corpus del saber que la antecede y desde el cual se generan las decisiones y las prácticas curativas de cada patología.

\section{OBESIDAD: CAUSA O EFECTO}

La obesidad ha sido tratada como causa de enfermedades cardiovasculares, metabólicas y endocrinas y por lo mismo múltiples estudios han estado destinados a reconocer los riesgos, la predisposición y los factores de protección. Se han invocado aspectos que van desde lo genético $(2,3)$ hasta lo ambiental, incluidos en estos el uso de la información nutricional por los mass-media. Desde este paradigma se han realizado campañas de nutrición saludable, ejercicios y prevenciones desde edad temprana en jardines de preescolares colegios y población general. Los resultados globales no son promisorios puesto que cada vez hay más obesos (4).

Necesariamente surge la inquietud de preguntarnos que es lo que no estamos reconociendo: ¿factor o dinámica de factores? ¿O es tal vez que el modo de conceptualizar la obesidad no nos permite pensarla -solucionarla desde una perspectiva distinta? 
Si tratamos la obesidad como causa de otras patologías se intentarán remover sus orígenes es decir el balance calórico positivo. Desde esta posición intentaremos que los pacientes: restrinjan calorías con dietas; se sometan a cirugías destinadas a disminuir el volumen del estomago; se aumentará su gasto calórico ya sea a través de medicamentos o ejercicios; se bajará la absorción de alimentos especialmente los grasos a través de drogas (bastante incomodas por sus efectos colaterales). Es decir, intentaremos modificar la ingesta, el ejercicio físico y sus externalidades.

Si en cambio tratáramos la obesidad como efecto, nos preguntaríamos acerca de la causa y esto nos llevaría a reconocer que algo ha ocurrido en la regulación de los procesos de ingesta. Estos se encuentran asociados tanto al hambre y la saciedad de regulación hipotalámica como a circuitos de origen y función emocional . Emoción e ingesta, así como emoción y actividad muscular parecen actuar conjuntamente. ¿Cómo podemos entender esta asociación?

\section{LAS BASES DE LA VIDA}

Los sistemas primarios de sobrevivencia son sistemas muy primitivos, propios de todos los seres vivos que necesitan incorporar energía, aprender del medio para sobrevivir en él y construir sociedad. Entre los circuitos que se consolidan muy tempranamente o que tal vez son dotación genética pura estarían: hambre, frío, stress, procreación, alarma, cuidado, territorialidad. (5) Estos circuitos pueden ser reconocidos y delimitados por su neurofisiología ( 6,7 ). Las estructuras nerviosas que participan en ellos pueden ser localizadas, las substancias que los movilizan individuadas e incluso los modos de modulación y la conducta que participan en ellos descritos y generalizados. La capacidad de modular los procedimientos de cada uno de estos sistemas supone aprendizaje, es decir, memoria de acoplamiento (8) entre el ser vivo y su medio ambiente.

Los sistemas de hambre / saciedad; alarma y procreación no son considerados circuitos emocionales aunque requieren de estos para lograr funcionar. Estarían más cerca de una dotación que podemos llamar instintiva que responde a estímulos reconocibles y universales entre la especie animal, por ejemplo si un animal grande ingresa a nuestro campo visual se encenderá la alarma y nuestra respuesta será arrancar, agredir o bloquearnos. Si por otra parte, la baja la glicemia, se estimulará el hambre y la búsqueda de alimentos. Con los estímulos sexuales se encenderán los circuitos de procreación.

Por otra parte, los circuitos emocionales aunque no tan evidentes como los anteriores, podemos delimitarlos tanto por las regiones cerebrales que utilizan como por las conductas que muestran. Son precisamente estos sistemas neurofisiológicos emocionales los que pueden actuar tanto como estimulador así como de inhibidor de las conductas asociadas al comer.

La modulación emocional es la tarea de los procedimientos psíquicos. Se entiende el "aparato psíquico" como un dispositivo que media es decir, usaremos una metáfora de nuestra era tecnológica, una especie de "driver" que permite que el sistema operativo pueda entenderse con periféricos a través de algún mecanismo que permita controlar su funcionamiento. Se trataría de un conjunto de instrucciones que indican la manera en que debe realizarse la interfaz. La psiquis media entre la biología del organismo y las circunstancias en que este se encuentra desplegando sus procesos vitales. Por eso el individuo no puede entenderse sin las circunstancias que lo intervienen y por eso las circunstancias se encuentran con las fronteras del organismo humano para producir conductas.

La psiquis es el modulador adquirido del ser vivo. El aprendizaje de esta modulación se inicia desde que se inicia la vida, almacenando información de procedimientos que se repiten en memorias fisiológicas que no siempre tienen representación mental y de las que por lo tanto, no siempre es posible dar cuenta consiente.

La psiquis administra diversos procedimientos cuya finalidad es conservar el equilibrio fisiológico, por ejemplo, a pesar que en la pantalla del televisor se aparece un león iracundo y con la boca abierta, la psiquis procesa la activación amigdaliana de peligro (9) activando circuitos inhibitorios (10) que se han desarrollado al comprender que una imagen no es la realidad. Por lo tanto, simultáneamente con la aparición de la imagen, se activan los circuitos de alarma y de inhibición de la alarma y podemos permanecer en frente de la pantalla sin taquicardia.

La sensación de control, que este acto provee, es una ganancia secundaria que para algunos se torna adictiva (adictos al control).

La psiquis utiliza como unidades de información básica las emociones y estas a su vez son catalogadas no solo desde el lenguaje sino a través de grupos de sensaciones que aprendemos a distinguir desde el cuerpo.

Los circuitos emocionales primarios se caracterizan por:

1. Tener un rango fisiológico especifico, asociado con un claro estimulo y respuesta universal expresado en la conducta y expresión facial.

2. Distinguir un trazado del circuito neuronal que utilizan 
3. Tener una combinación particular neuroquímica

4. Ser de base genética y su función se puede alterar por mutaciones en genes específicos

5. Tener un origen posible de entender a través de uso para necesidad de sobrevivencia

6. Ser universales en humanos y homólogos a otras especies. Tener precursores evolucionarios

7. Su disfunción puede asociarse a trastornos psiquiátricos. Ya sea por aumento o disminución de sus funciones.

Entre las funciones de los circuitos emocionales primarios están:

1. Emociones básicas: a. Placer b. Búsqueda c. Asco d. Rabia e. Miedo

2. Reproducción: Deseo

3. Filiación: Apego

4. Regulación de conflicto: a. territorialidad b. Jerarquías: Dominio y sumisión

5. Aprendizaje: Juegos de imitación y competencia.

Los circuitos emocionales primarios participan en la conducta del comer cuando la psiquis no direcciona bien la información emocional, no procesa sus contenidos y lo desvía a sensaciones de ansiedad que los pacientes solucionan comiendo. Esta "ansiedad" suele ser un grupo de afectos desagradables entre los que se encuentra la rabia, la tristeza, el aburrimiento, el vacío. La solución de comer en vez de elaborar estas sensaciones indica que existen ciertas zonas psíquicas no desarrolladas.

Se entiende este silencio psíquico como un mecanismo de defensa muy precoz que no ha permitido articular los niveles de la experiencia con la conciencia posiblemente secundario a traumas (cantidades de cortizol muy elevados en un momento del desarrollo) (11).

\section{MÁS ALLÁ DE LA ALIMENTACIÓN: EL COMER}

Comer es una conducta que relaciona diversas dimensiones. La participación de los circuitos emocionales forma parte de la red que asegura la inclusión de energía al organismo.

Tomando como modelo los circuitos mencionados podemos decir que en el comer psíquico participarían estas vías desplazando sus acciones hacia la ingesta o el rechazo a la misma de modo que tanto el placer como la búsqueda quedarían fijadas en la alimentación. El asco (circuito de seguridad frente a lo venenoso) podría desplazarse hacia el rechazo no solo de alimentos sino de situaciones o personas desagradables fijando esta función en la comida (mecanismo utilizado en la restricción alimentaria de las anorécticas).

El deseo, que es un circuito destinado a la búsqueda del apareamiento, podría desplazar y cambiar el objeto sexual por sensaciones digestivas. El apego, que es un sistema de protección relacional a través del cual la cercanía con el cuidador regula significativamente el bienestar, puede intercambiar alimento por cuidador. La territorialidad, que es un sistema a través del cual se delimita el espacio de dominancia e individualidad, puede ser utilizada preferentemente en la fijación de la cantidad y variedad de comida con las evidentes consecuencias en la conducta de comer.

Todos estos procedimientos cercanos al comer son utilizados normalmente, es la rigidez o la fijación de algunos de estos circuitos lo que define la patología del comer.

Podemos simplificar diciendo que el comer se encuentra asociado con los circuitos primarios emocionales del siguiente modo:

\begin{tabular}{|l|l|}
\hline \multicolumn{1}{|c|}{$\begin{array}{c}\text { Características } \\
\text { del comer }\end{array}$} & \multicolumn{1}{c|}{ Circuito primario } \\
\hline Estimulación del comer: & Placer. \\
\hline Inhibición del comer: & Asco. \\
\hline $\begin{array}{l}\text { Estimula o inhibe } \\
\text { según aprendizaje } \\
\text { y circunstancias } \\
\text { juego. }\end{array}$ & $\begin{array}{l}\text { Búsqueda, rabia, miedo; } \\
\text { apego; deseo; } \\
\text { territorialidad, jerarquía, }\end{array}$ \\
\hline
\end{tabular}

La selección de funciones que estimulen o inhiban el comer depende tanto de la historia familiar como de los eventos emocionales a que ese organismo haya estado sometido. Por esto para llegar a desarticular los procedimientos se requiere comprender y producir cambios en la configuración vital de la persona.

Comer es tan importante que no es posible sobrevivir por largo tiempo si no hay ingesta suficiente. Los pacientes con anorexia comen, pero no suficiente y las cifras de mortalidad en esta población son más elevadas que en la población general $(12,13)$. Son organismos sometidos a stress nutricional que se complican a lo largo de su enfermedad con patología cardiaca, hepática (especialmente si consumen alcohol en forma excesiva) (14) renal, metabólicas y finalmente mentales (que las pueden llevar incluso al suicidio.

Cuidar que el circuito ingesta permanezca operante es por lo tanto esencial para la especie. La ingesta requiere básicamente de un ambiente que la permita: disponibilidad de alimentos; pertinencia de consumirlos; territorialidad; alertas en la huida de otros en competen- 
cia. Sin embargo, la (15) evaluación del ambiente en la especie humana se realiza a través de la psiquis de modo que ésta opera con sus lógicas asignando significado tanto a la experiencia con el ambiente cuanto a la experiencia interna del organismo. Estos significados se construyen psíquicamente es decir transformando la percepción de la vivencia en estado de la realidad. La actividad psíquica es esencialmente transformacional, es decir convierte, modifican y deforman la realidad hasta hacerla aceptable al individuo o la sociedad desde donde surgen sin alterar su funcionamiento fisiológico.

Alimentarse por tanto quedará incluido dentro de la conducta del comer y está dentro de las lógicas psíquicas. Comer es una conducta de relación con otros, consigo mismo y con los alimentos. Se come haciendo ingesta, es decir introduciendo alimentos a la boca tragando y llenando el estomago. Se come haciendo rituales, corporizando una cultura, compartiéndola con otros. Cualquiera de estos pasos puede ser deformado por la psiquis buscando regulaciones extra alimentación y aumentando el riesgo de algún trastorno en la conducta del comer.

\section{ACUMULANDO ENERGÍA SIN LOGRAR HOMEOSTASIS}

Nuestra civilización ha logrado disponibilidad de alimentos a través de técnicas de conservación, distribución y mercado lo que ha posibilitado su uso para fines mas allá de lo nutricional. Hay quienes se oponen a consumirla (anorexia nervosa), o si la consumen se oponen a utilizarla y la rechazan (bulimia nervosa), mientras otros no paran la ingesta o no utilizan la energía ingresada y desarrollan obesidad.

El balance energético positivo por lo tanto, se puede deber básicamente a:

- Aumento de la ingesta y por defecto suponemos que la ingesta está cumpliendo con funciones que no son alimentarias.

- Disminución de la necesidad de energía y por defecto suponemos que el sujeto se está moviendo menos o bajó su gasto calórico basal.

Ambas condiciones pueden corresponder a desregulaciones fisiológicas o dicho de otro modo alteraciones psíquicas en las funciones de regulación del organismo.

\section{ALTERACIONES EN LA CONDUCTA DEL COMER}

Las desregulaciones de la psiquis producen conductas, signos y síntomas que se hacen evidentes al ocurrir la consecuencia que es el aumento de peso. Podemos separar en tres categorías estas desregulaciones: 1-Comer en vez de elaborar psíquicamente la realidad; 2-comer para obtener placer; 3 -comer como parte de un modo de estar con otros.

Estas deregulaciones las llamaremos comer psíquico

\begin{tabular}{|l|l|}
\hline \multicolumn{1}{|c|}{$\begin{array}{c}\text { Funciones del } \\
\text { comer psíquico }\end{array}$} & \multicolumn{1}{c|}{ Suplanta a } \\
\hline $\begin{array}{l}\text { Evitación del trabajo } \\
\text { de elaboración psíquica } \\
\text { que construye significado } \\
\text { funcional de la realidad. }\end{array}$ & $\begin{array}{l}\text { Definición de límites del } \\
\text { yo (abandono de } \\
\text { omnipotencia infantil) } \\
\text { Aprendizaje de negocia- } \\
\text { ciones intersubjetivas } \\
\text { (yo en relación a otro })\end{array}$ \\
\hline Búsqueda de placer básico & $\begin{array}{l}\text { Diversidad y desarrollo } \\
\text { de vías sublimadas } \\
\text { (elaboradas por la } \\
\text { conciencia) }\end{array}$ \\
\hline $\begin{array}{l}\text { Pertenencia básica a } \\
\text { grupo de filiación. }\end{array}$ & Autonomía \\
\hline
\end{tabular}

\section{1- Comer en vez de elaborar psíquicamente la realidad}

Las desregulaciones emocionales utilizan el comer como función psíquica, y la psiquis no hace su trabajo de evaluación, integración, sentido.

Cada uno de los eventos o estados descritos a continuación son capaces de sostener un tipo de ingesta que podemos nominar como ingesta psíquica que ocurre sin fines nutricionales, sino en búsqueda de equilibrios que la psiquis no pudo solucionar.

Es decir la persona suplanta la actividad psíquica necesaria para resolver la dificultad emocional con alteraciones en la conducta del comer. Estas alteraciones implican diversas conductas como: aumento de la ingesta; picoteo; craving a hidratos de carbono; supresión de saciedad; atracones.

1. Anticipación de amenaza a la homeostasis: Futuros cambios: de casa, colegio, trabajo, dependencia laboral.

2. Separaciones de la figura de apego: cambios sutiles en la distancia emocional con la figura de apego principal (madre, padre o pareja)

3. Vacío, aburrimiento.

4. Ansiedad anticipatorio ante evaluaciones o juicios de terceros

5. Sensación de inhabilidad para el aprendizaje de tareas específicas

6. Ser individuo de características dominante en 
posición de sumisión

7. Ser individuo con características de sumisión y estar en posición dominante

8. Amenza al territorio propio. (concreto o simbólico)

9. Desafiliación (dejar de pertenecer a un grupo de referencia)

10. Desmotivación: incluido el deseo sexual.

\section{2-Comer para obtener placer}

Desregulaciones en el circuito de placer de la ingesta (ABUSO DE INGESTA)

Este modo de utilizar circuitos del placer para aumentar la ingesta se parece al estilo alcohólico donde el alimento (muchas veces hidratos de carbono en presentaciones dulces) activan el circuito y lo dejan reverberando, buscando mas y mas estimulación. Los pacientes paran, lo mismo que el alcohólico cuando ya no pueden más de volumen o cuando se acaba el alimento. Este Craving por sustancias calóricas es muy difícil de parar y solo responde a medidas de control que toman parientes o el paciente mismo.

La similitud entre la ingesta de alimentos y la ingesta de drogas se puede ver en tabla anexa (adecuada de 16)
Cuando el paciente es un abusador de alimentos y está fijado en este circuito puede aparecer como un obeso, bulímico o anoréctico según regule los mecanismos de control del craving tendrá más o menos éxito en el control del peso.

En este tipo de pacientes las dietas con "licencias" (haga dieta en la semana y tiene permiso el fin de semana) funcionan siguiendo la lógica y el ritmo atracón/ restricción y (control/descontrol) que es justamente lo enfermo y que se suelen acompañar de variaciones en el ánimo y la angustia.

Parar este tipo de ingesta los medicamentos del tipo IRSS puede ser de ayuda. (17).

\section{3- COMER COMO PARTE DE UN MODO- DE- ESTAR- CON- OTROS.}

Desregulaciones psíquicas producidas en campos vinculares adversos (relaciones conflictivas abiertas u ocultas)

Las relaciones significativas son aquellas capaces de regular la homeostasis a través de la presencia o ausencia del cuerpo designado como significativo. Una relación tiene significación cuando es capaz de producir cambios fisiológicos en el organismo. Generalmente esto ocurre en las relaciones que llamamos de mayor intimidad (pareja, hijos, padres). Se llama campo vincular a

\begin{tabular}{|c|c|c|}
\hline $\begin{array}{l}\text { Criterio utilizado en } \\
\text { abuso de drogas }\end{array}$ & Características & $\begin{array}{l}\text { Propuesta criterio en } \\
\text { abuso de ingesta }\end{array}$ \\
\hline Tolerancia & $\begin{array}{l}\text { Cantidad necesaria para lograr sa- } \\
\text { tisfacción. }\end{array}$ & $\begin{array}{l}\text { La saciedad se alcanza con cantidades } \\
\text { crecientes de comida }\end{array}$ \\
\hline Síntomas de privación & $\begin{array}{l}\text { Desequilibrio fisiológico producido } \\
\text { por la falta de una sustancia especi- } \\
\text { fica exógena. }\end{array}$ & $\begin{array}{l}\text { Disforia y distress cuando no hay dis- } \\
\text { ponibilidad de alimentos }\end{array}$ \\
\hline Compulsión & $\begin{array}{l}\text { Consumir aunque sea mas allá de } \\
\text { lo deseado. }\end{array}$ & Comer mas de lo deseado. \\
\hline Descontrol & $\begin{array}{l}\text { Incapacidad de cortar el consumo } \\
\text { una vez iniciado. }\end{array}$ & $\begin{array}{l}\text { Intentos fallidos de control en la ali- } \\
\text { mentación. }\end{array}$ \\
\hline Invasión & $\begin{array}{l}\text { Irrupción de impulsos pensamientos } \\
\text { y conductas destinadas a conseguir, } \\
\text { utilizar y recuperarse del uso de } \\
\text { sustancia. }\end{array}$ & $\begin{array}{l}\text { Gran porcentaje del tiempo diario usado } \\
\text { en el tema del comer: recetas, fantasías } \\
\text { de comidas, búsqueda y compra de } \\
\text { comida, ingesta y recuperación de } \\
\text { ingesta. }\end{array}$ \\
\hline Aislamiento social & $\begin{array}{l}\text { Reducción de la actividad social y } \\
\text { cambio por actividad de ingesta. }\end{array}$ & $\begin{array}{l}\text { Reducción o suspensión de vida social } \\
\text { a causa del consumo de alimentos y o } \\
\text { sus consecuencias. }\end{array}$ \\
\hline Reincidencia & $\begin{array}{l}\text { Repetición de conducta a pesar de } \\
\text { consecuencias. }\end{array}$ & $\begin{array}{l}\text { El consumo se mantiene a pesar de las } \\
\text { consecuencias físicas y psíquicas. }\end{array}$ \\
\hline
\end{tabular}


la regulación mutua entre dos o más cuerpos necesaria para sostener el bienestar común. Este campo vincular está caracterizado por un cierto clima emocional que ha puesto en acción ajustes emocionales de cada miembro destinados a sostener la sintonía emocional entre ambos. Estos ajustes implican al organismo en su totalidad y suelen ser muy inconscientes. Campos vinculares donde la fisiología queda ajustada de un modo que podemos señalar al menos como incómodo o desagradable predisponen a buscar el comer como forma regulación. Dado que comer y ser cuidado (aliviado) son estados que al inicio de la vida se dan juntos y fusionados (18) se confunde la regulación emocional con comer y se come en vez de regular.

La coordinación y negociación de las emociones que se activarán en el campo vincular requiere de acuerdos activos y muy rápidos que suceden sin mediar lenguaje y en los cuales el comer pudo quedar capturado como parte del estar- junto- a -otro.

Como la sintonía relacional está expuesta a quiebres y discontinuidades $(19,20)$ el comer puede ser utilizado como reparador del clima base. Comer reconstituiría la sintonía, aunque a la larga junta kilos de más.

Circuitos cerebrales implicados en la regulación del consumo de alimentos: $(21,22,16)$.

\begin{tabular}{|l|l|}
\hline \multicolumn{1}{|c|}{ Funcion Alterada } & Zona Cerebral Implicada \\
\hline $\begin{array}{l}\text { Control inhibitorio al } \\
\text { impulso de consumo. }\end{array}$ & $\begin{array}{l}\text { Corteza prefrontal; } \\
\text { giro cingulado anterior. }\end{array}$ \\
\hline $\begin{array}{l}\text { Atención preferente } \\
\text { al alimento. }\end{array}$ & $\begin{array}{l}\text { Núcleo accumbens, } \\
\text { hipotalamo, pallido. }\end{array}$ \\
\hline $\begin{array}{l}\text { Hábitos condicionados } \\
\text { a comer en vez de } \\
\text { enfrentar conflicto }\end{array}$ & $\begin{array}{l}\text { Amígdala, hipocampo, } \\
\text { estriatum dorsal }\end{array}$ \\
\hline $\begin{array}{l}\text { Alta motivación a } \\
\text { consumir alimentos }\end{array}$ & $\begin{array}{l}\text { Corteza orbitofrontal, } \\
\text { núcleos mesencefálicos } \\
\text { dopaminergicos }\end{array}$ \\
\hline
\end{tabular}

\section{CONCLUSIÓN}

Si pensamos la obesidad como la consecuencia de un intento que hace el organismo por lograr su homeostasis a través del consumo de alimentos, entonces la mirada se traslada hacia el psiquismo, el encargado de conservarla.

Algo no estaría funcionando bien en los sistemas de regulación emocional que se recurre más veces al comer que a mecanismos psíquicos de auotoregulación o regulación emocional.

El placer de comer, por ejemplo, sustituiría o enmascararía dificultades en la motivación, la ingesta podría ser utilizada como un símil materno (consuelo, apoyo, protección, sostén) o como prótesis en los derrumbes emocionales.

Estos circuitos básicos pueden por momentos suplir actividad psíquica y esto es considerado normal, sin embargo si quedan frecuentemente o completamente a cargo de regular el acontecer emocional y por lo tanto fisiológico del organismo humano, (23) las consecuencias serán el sobrepeso y la obesidad.

La intervención de los sistemas que regulan los desequilibrios y rupturas del bienestar fisiológico permitiría liberar de esta sobreactividad a las zonas destinadas a la ingesta y la sobreviviencia.

El bienestar fisiológico es una sensación de tranquilidad que implica el mejor funcionamiento de ese organismo, es decir, el mejor acoplamiento a las circunstancias donde se encuentra desplegando sus capacidades vitales.

La continuidad de este bienestar está vigilada por el aparato psíquico quien utilizando múltiples mecanismos (distorsionar, desplazar, negar percepciones, racionalizar, proyectar sensaciones desagradables y otros) logra sostener una homeostasis sana.

El desarrollo de un aparato psíquico capaz de cuidar la continuidad de la homeostasis ocurre desde el nacimiento a través del aprendizaje por acoplamiento fisiológico con los cuidadores. Por esta razón el estado emocional de los cuidadores es tan importante para lograr un campo de interacción fisiológica al que llamamos vínculo.

Intervenciones en el vínculo, lugar de aprendizaje de la regulación emocional, parecen augurar mejores resultados en la prevención de la obesidad, lo mismo que, ya producidos mecanismos desregulados la reparación de los mismos a través de intervenciones terapéuticas. (24)

Tal vez ha llegado el momento de pensar la obesidad como una consecuencia de una disfuncionalidad en la homeostasis del organismo secundaria a un psiquismo dañado en mayor o menor extensión y no solo como la causa de enfermedades orgánicas. ¿Será necesario incluirla en el DSM-IV considerando que son los componentes mentales la clave para su tratamiento?

\section{RESUMEN}

Se propone ampliar la mirada de la obesidad hacia la relación ingesta-vida emocional. Se analizan las regiones cerebrales que participan compartiendo circuitos primarios en ambas situaciones. Se propone el comer más allá de la alimentación con funciones mentales como: auto-regulación emocional; búsqueda de placer y participación en el ajuste emocional de las relaciones significativas (familia, pareja). Se sugiere que la 
inclusión de la obesidad en el Manual de enfermedades mentales cambiaría su comprensión ampliando las alternativas para su prevención y tratamiento.

Palabras claves: obesidad, enfermedad mental, desregulación emocional, DSM.

Dirigir la correspondencia a:

Dra.

Patricia Cordella M.

Unidad Trastornos de Alimentación

Departamento de Psiquiatría

Pontificia Universidad Católica de Chile

Teléfono: 2205455

La capitanía 976

E-mail: mpcordella@gmail.com

\section{BIBLIOGRAFÍA}

1. American Psychiatric Association. Diagnostic and Statistical Manual of Mental Disorders, Fourth edition (DSM IV). Washington DC, APA 1994.

2. Bulik C, C.T Slof-Op't Landt, Van Furth E, Sullivan P. The genetics of Anorexia Nervosa Annual Review Nutr 2007; 27:263-275.

3. Ribase's a,b M, Fernández-Aranda F, Grataco M, Mercader J. Contribution of the serotoninergic system to anxious and depressive traits that may be partially responsible for the phonotypical variability of bulimia nervosa. J Psychiatric Res 2008; 42: 50-57.

4. Encuesta Nacional de Salud (ENS). Ministerio de Salud. Chile. 2003.

5. Pankseep J. Emotional endotypes in evolutionary psychiatry. Progress in Neuro pharmacology and biological Psychiatry 2006; 30 :774-784.

6. Godart N, Flament M, Perdereau F, Jeammet P. Co morbidity between eating disorders and anxiety disorders: a review. Int J Eat Disord 2002;32:25370.

7. Kaye W, Strober M, Jimerson D. The neurobiology of eating disorders. In The Neurobiology of Mental Illness, ed. D Charney, E Nestler, New York: Oxford Univ. Press 2004, pp. 1112-28.

8. Maturana H, Varela F. El árbol del conocimiento. Ed Universitaria, Santiago de Chile, 1989.

9. Davis M. Are different parts of the extended amygdale involved in fear versus anxiety?. Biol Psychiatry 1998; 44: 1239-1247.

10. Mc Ewen B, Milner T. Hippocampal formation: shedding light on the influence of sex and stress on the brain. Brain Res Rev 2007; 5:343-355.

11. Mc Ewen B. Neurobiol Aging 2002; 23 (5) : 921939.

12. K Theander S. Standardized mortality in eating disorders: a quantitative summary of previously published and new evidence. J Psychosom Res 1998; 44: 413-34.

13. Crisp AH, Callender JS, Halek C, Hsu LKG. Longterm mortality in anorexia nervosa: a 20-year follow-up of the St George's and Aberdeen cohorts. Br J Psychiatry 1992; 161:104-107.

14. Bulik CM, Klump KL, Thornton L, et al. Alcohol use disorder co morbidity in eating disorders: a multicenter study. J Clin Psychiatry 2004;65:1000-6.

15. Graybiel AM. Habits, Rituals, and the evaluative brain. Annual Review Neuroscience 2008; 31: 359-387.

16. Volkow N, O’ Brien C. Issues for DSM-V: Should obesity be included as a brain disorder? Editorial Am J Psychiatry 2007; 164(5): 708-710.

17. Yager Y. Practice guideline Am Psychiatric Association. for the treatment of patients with eating disorders (Revision). Am J Psychiat 2000; 157 Suppl 1: 1-39.

18. Cordella M Patricia. Proposición de un modelo para comprender la configuración anoréctica. Rev Chil Pediatr 2002;73:566-575.

19. Schore A. Advances in neuropsychoanalysis, attachment theory, and trauma research: Implications for self psychology. Psychoanal Inquiry 2002 ; 22: 433-484.

20. Gunnar M, Quevedo K. The Neurobiology of Stress and Development Annu Rev Psychol 2007;58:145173.

21. Uher R, Murphy T, Brammer MJ, Dalgleish T, Phillips ML, Ng VW, Andrew CM, et al. Medial prefrontal cortex activity associated with symptom provocation in eating disorders. Am J Psychiatry $2004 ; 161: 1238-1246$.

22. Ward A, Ramsay R, Treasure J. Attachment research of eating disorders Brit J Med Psychol 2000; 3:3551.

23. Pally R. Emotional Processing: The Mind-Body Connection. Internat J Psycho-Analysis 1998; 78, 349-362.

24. Southgate L, Tchanturia K, Treasure J. Buliding a model of the aethiology of eating disorders by translating experimental neuroscience into clinical practice. J Mental Health 2005; 14(6): 553 - 566. 